\title{
The professional and organizational future of imaging
}

\author{
ESR Executive Council 2009 • European Society of Radiology
}

Received: 23 October 2009 / Accepted: 23 November 2009 / Published online: 28 January 2010

(C) The Author(s) 2010. This article is published with open access at Springerlink.com

\section{Introduction}

The field of biomedical imaging and particularly the specialty of radiology have expanded dramatically over the last decade. The current role of the radiologist is being challenged as use of images by clinicians is becoming increasingly important. Some of the main challenges radiology faces are due to the increasing workload and subsequent shortage of radiologists, the increasing availability of technologies allowing partial outsourcing of imaging services (teleradiology), the ease of the use of images and therefore an increased ability of knowledgeable clinicians to read them. Moreover, control of patients is central to turf wars, and here radiologists are at a disadvantage in comparison with other clinical specialists. Thus according to William R. Hendee "radiology is vulnerable to the erosion of its domain of expertise because of its dependence on referrals from other physicians" [1].

Pierre Schnyder, head of radiology at CHUV in Lausanne (Switzerland), described self-referral politics as representing the cornerstone of turf wars [2], with interventional radiology being especially at risk. The possibility of controlling specific imaging units or imaging technologies offers an attractive alternative for selfreferring clinicians, but it may stimulate detrimental competition for patients, space, and resources. Moreover, internal competition among specialists interested in imaging may result in a deterioration of normally collegial relationships. There is also evidence that self-referral of

ESR Executive Council $2009 \cdot$ European Society of Radiology $(\varangle)$ Neutorgasse 9/2,

1010 Vienna, Austria

e-mail: communications@myESR.org imaging services often leads to overuse of services and creates unjustified health care expenses [1]. It is also suggested that "the accuracy of imaging procedures is compromised because many self-referring physicians do not have any comparable imaging training or expertise" [1]. Additional knowledge in understanding the acquisition and display processes of medical images is not something that can be acquired as a sideline to the practice of another medical specialty.

Preserving the integrity of radiology as a specialty also has additional advantages. Alexander R. Margulis, former head of radiology at UC San Francisco, suggested that fragmentation has a negative effect in that it separates those outside the imaging specialty from advances in the general field, removes them from cooperation with other radiologists and basic scientists in imaging, and usually makes them too one-sided and less valuable to patients [3]. Moreover, "every imaging examination exposes patients to some element of risk. That risk comes from unwarranted exposure to radiation, as well as from false-positive or false-negative examination results. Knowledge about why, when, where, how, and for whom imaging should be employed" [1] is an integral part of radiologists' training and constitutes the quality and safety standards of radiological services.

From an organizational point of view, customers value and need a comprehensive practice of imaging delivered "24/365" (all day, every day). The workflow of such a service includes quality assurance for professional and technical staff, monitoring of the appropriateness of referral, report generation, archiving, and last but not least supervision and consultation by highly trained superspecialized radiologists [4]. However, an integrated comprehensive imaging service does not exclude the possibility 
of decentralizing some of the facilities (equipment) for the sake of patient-centered care despite some wastefulness and inefficiency.

Solutions for most of the problems clearly underscore the advantages of a comprehensive and integrated approach for the domain of diagnostic imaging and image-guided interventions, with a frequently beneficial and needed collaboration with clinical specialties on an equal basis. However, the integrity of the organizational structure as a centralized "imaging facility" within the enterprise bears numerous benefits for cost containment, quality standards, and efficiency. Limited decentralized services are delivered upon mutual local agreement between departments.

\section{Erosion and fragmentation of radiology as a specialty}

From the beginning, radiology has been vulnerable to the erosion of its domain of expertise because of its dependence on referrals from other physicians. This vulnerability makes radiology susceptible to efforts by other physicians to provide their own imaging services and not to refer patients to radiologists [1]. There is an excess of physicians in some specialties, and nonradiologist physicians have become more familiar with the imaging techniques most frequently employed in their practices. Also academic careers are frequently based on controlling the latest new technology. Therefore it is quite common in large enterprises that certain imaging procedures and/or technologies are available multiple times with all the consequences of internal competition, inefficient use, different workflows, uncoordinated capital investment, various standards of referral, execution and interpretation, etc.

Fragmentation of the specialty, in contrast, represents a separation or break-away of some parts of the imaging services from the original organizational entity, i.e., the department of radiology. The reasons for fragmentation can be explained by the dramatic expansion of the domain of activity of radiologists. No single person can master all the available knowledge. The response to this development has been a gradual increase in the degree of subspecialization of radiologists. Initially subspecialization occurred based on different modalities in the 1970s and 1980s, while later a organ-system-based subspecialization became much more appropriate and was gradually adopted both in the structure of radiological services as well as in the training curriculum [5]. Subspecialization is a clear need for radiologists in large academic and community hospitals and increasingly even in private practices. However, in most of cases subspecialty sections remain within the overarching department of radiology with the benefit of shared facilities, efficient use of resources, and common organizational structures.
Typically, a fragmentation occurred in Europe with nuclear medicine. Whereas in the United States, nuclear medicine remained a subspecialty of radiology and mostly part of radiology departments, it became a separate medical specialty and frequently a totally autonomous organizational entity (department) in Europe [3]. In some parts of Europe, neuroradiology is increasingly trying to separate itself from departments of diagnostic radiology [6]. Something similar could happen with other subspecialties of diagnostic radiology such as cardiovascular, pediatric, or orthopedic imaging as well as with imageguided interventions [6].

Fragmentation has a negative effect in that it separates those involved in only parts of the profession from advances in the general field of imaging, removes them from cooperation with other radiologists and basic scientists in imaging, and usually makes them too one-sided and less valuable to patients [3]. A very good example is the advent of hybrid imaging techniques (PET-CT, SPECTCT, MR-PET) in which collaboration of nuclear physicians and radiologists trained in different parts of the technology is needed, leading to inefficiency, delays, and higher costs.

\section{Turf wars in radiology}

A search for "turf wars" and "radiology" on the Internet will return more than 100,000 hits. These so-called wars have been prompted by advances in imaging that have drawn other specialists to the turf of the radiology department, most notably those in cardiovascular medicine. Boundaries are being crossed, and conflict and competition have become inevitable [7].

As stated by Schnyder, "theoretically and ideally, clinicians and radiologists should work side by side in order to provide patients with the best standards of care. Practically, on a ground of increasing financial restrictions, struggles between nonradiologists and radiologists are mostly related to financial considerations, power and, not infrequently, to personal fame." The possibility of controlling radiology units or radiological technologies offers an attractive alternative for self-referring clinicians [2]. Control of patients is central to turf wars, and here radiologists, particularly diagnostic radiologists, are at a disadvantage in comparison with clinical specialists [8].

Additionally, during the mid-1960s and early 1970s, radiologists frequently showed a lack of foresight or limited interest in new technologies, such as echocardiography and obstetrical ultrasonography, catheter coronary angiography, and ERCP, thus leaving an open door to internists, pediatricians, obstetricians, gynecologists, cardiologists, and gastroenterologists [2]. "Some of these fields of 
diagnostic imaging were lost without any struggle and probably forever" [2].

With regard to the issue of "turf wars" among physicians over cardiac imaging procedures, Anthony N. DeMaria, an eminent cardiologist and Editor-in-Chief of the Journal of the American College of Cardiology, stated: "I believe that the optimal provision of these services will require both cardiologic and radiologic skills. This can be achieved by collaboration or by the acquisition of cardiologic skills by radiologists or vice versa. The individuals who will own the imaging turf will be those who have broad-based skills in all aspects of cardiology and imaging" [9].

\section{Turf wars in image-guided interventions}

There is little doubt that current interventional procedures and future developments in image-guided interventions will dominate treatment in medicine [10]. This is a result of the intrinsic advantage over open surgical approaches: the minimally invasive character of the procedures. Whereas the basis of interventional radiology in the future is not in question, the domain of the procedures may be: will interventional radiologists continue to perform the majority of interventional radiology procedures $[6,11]$ ? There has been a huge amount of discussion of turf wars by and among many specialties, and interventional radiology is especially challenged, with many of the procedures being increasingly performed by nonradiologists (e.g., noncoronary angioplasty and stent placement) [12-14].

Endovascular surgery applies to manipulative diagnostic and therapeutic procedures carried out through and within blood vessels. Many of the techniques involved are catheter-guidewire-based and use fluoroscopic or ultrasonographic imaging modalities for control. Access to the vascular tree for endovascular manipulative treatment can be gained via percutaneous puncture utilizing Seldinger wire-catheter-sheath technology or, less commonly, by open exposure and arteriotomy or venotomy. Endovascular treatment techniques have already changed the way arterial and venous diseases are managed. In addition, it is likely that these techniques will have an even greater influence on the way vascular diseases are managed in the future [15]. According to Veith at al., because these intraluminal therapies are a simpler, safer way to treat some vascular disease patients and because their less invasive nature renders them attractive to referring physicians and patients alike, endovascular techniques have been embraced by vascular surgeons and used increasingly by them. In some cases, these catheter-based treatments have been used directly by the vascular surgeon; in other circumstances, management has been by collaboration with the interventional radiologist [15].
Thus the role of interventional radiologists in percutaneous peripheral vascular interventions is currently being challenged in some hospitals by vascular surgeons and cardiologists $[6,13]$. It is worth considering published remarks made by some leaders of these specialties with regard to the degree of training they receive in such interventions. For example, J.M. Porter, a leading vascular surgeon, made the following comment: "Clearly, these procedures [angioplasty and associated interventions] are based on arteriographic techniques including percutaneous catheterization, the use of contrast media, and ionizing radiation. I note that to become a highly skilled arteriographer requires three years of radiology residency, usually two years of angiography fellowship, and another two to three years of high volume clinical experience. To expect even a reasonably dexterous vascular surgeon or surgical resident to acquire similar skills in a few weeks or months is nonsense" $[16,17]$.

More recently F.J. Veith and M.L. Marin, also wellknown vascular surgeons, discussed the possible threat that transluminal endovascular graft placement poses to the practice of vascular surgery [18]. They stated, "These possibilities have already caused vascular surgeons and interventional radiologists to become concerned ... [T] he effective use of these endovascular technologies, though often requiring vascular surgical skills, always require catheter/guidewire-imaging skills, which many vascular surgeons do not possess" [13, 18].

\section{Additional challenges for the specialty of radiology}

Some additional challenges of the profession may have an impact on the outcome of the "turf wars" and therefore facilitate the undesirable erosion and fragmentation of imaging [6]:

- Rapid rise in workload and complexity of examinations have resulted in a shortage of radiologists in many countries, which may unfortunately result in clinicians undertaking the interpretations themselves. This is particularly the case in 24/7 emergency care and oncall provision.

- In most European countries, radiologists at present are satisfied with their overall position within the healthcare system and have no difficulties in finding professionally fulfilling and well-paid employment.

- The availability of high-speed internet transfer of images may result in centralized interpreting facilities which may affect the siting of radiological imaging equipment and the requirement for local radiologists.

- Improved image clarity and tissue differentiation in a number of situations have increased the ability of a 
knowledgeable clinician specialist to read his or her clinical diagnosis into the images, and often that diagnosis will be correct [3].

- The advent of molecular imaging has brought the need for large-scale multidisciplinary collaboration with basic scientists knowledgeable in molecular and cellular biology, nanotechnology, probe development, image processing, etc.

\section{Advantages of preserving the integrity of radiology as a specialty}

Radiology as a distinct specialty and organizational entity provides some major advantages regarding imaging procedures compared with other medical specialties [1].

\section{Referral}

Radiology is a referral specialty. With few exceptions, the radiologist depends on other physicians for requests for imaging examinations. This historical mode of practice is under threat. As described before, there are potent incentives for nonradiologists to take on imaging technologies and "self-refer" for imaging procedures rather than sending their patients to a radiologist $[8,19]$.

There is evidence that self-referral of imaging services is often economically motivated, leads to overuse of services, and creates unjustified health care expenses that are borne by health care payers and, ultimately, by patients $[1,8]$. It is also suggested that "the accuracy of imaging procedures is compromised because many self-referring physicians do not have the imaging training or expertise either to provide procedural and interpretive services at the same level of quality as do radiologists or to detect abnormalities other than those specific to their specialty. This reduction in accuracy puts patients at risk, increases the cost of health care, and erodes the public's confidence in the quality and safety of imaging services" [1].

Self-referral politics represents the cornerstone of turf wars, since radiologists, having no beds and no consultations, are unable to self-refer patients [2]. In 1990 B.J. Hillman et al., using a large private-insurance database, evaluated a series of 65,517 episodes of patients treated by 6,419 physicians for acute upper respiratory symptoms, pregnancy, low-back pain, or prostatism, and analyzed the corresponding imaging modalities, which included chest Xray, obstetrical ultrasound, lumbar spine radiographs, intravenous pyelograms, cystography, and ultrasound [20]. This paper strikingly demonstrated that physicians practicing self-referral requested 4 to 4.5 times more imaging examinations than physicians referring patients to radiologists $[2,20]$. The same study showed that resulting charges per patient were 4.4 to 7.5 times higher for selfreferring physicians [20]. In aggregate, these data raised the specter of overutilization related to the incentive for inappropriate self referral [9].

Knowledge in image interpretation

Residents in radiology have much to learn because the specialty is defined both by imaging technologies and by procedures that are becoming more numerous and sophisticated. According to Hendee, "to master all of these technologies and procedures, as well as to become expert in one or more, requires 5 years of intense residency training and, for most radiologists, additional years in organ-oriented sub-specialty fellowships" [1]. This amount of training, however, provides only a foundation to practice the radiologic specialty; a continuous expansion and refinement of the knowledge of the radiologist is required over the duration of practice [1]. Hendee goes even further by stating that "the development of knowledge in the acquisition and interpretation of clinical images is a fulltime effort" [1]. Knowledge in medical imaging is not something that can be acquired as a sideline to the practice of another medical specialty. Thus, it is understandable that the knowledge of radiologists is superior to that of other specialists who perform imaging and interpret images $[1,6]$.

\section{Broad clinical perspective}

A number of disorders may not be confined to one organ system, and there may also be circumstances in which the patient imaging examination identifies other abnormalities that were unsuspected and potentially life-threatening or unrelated to the symptoms being investigated. In these circumstances the radiologist-having a broad perspective and a wide knowledge of anatomy, pathology, and imaging signs - delivers an added value compared to a subspecialized clinician. It is important that there is good oversight to avoid the patient having unnecessary examinations and being referred to a variety of other physicians [6].

\section{Technology mastery}

Medical imaging employs highly complex technologies that are increasingly driven by the sophisticated computer and image-processing systems that are used for the acquisition and display of imaging data for interpretation. Understanding the acquisition and display processes and having a working knowledge of the complex interactions among these processes helps to ensure that optimal images are acquired for the medical conditions being investigated and that the images depict pathologic conditions in the patient 
and not errors in the processes of acquiring, manipulating, and displaying imaging data [1]. Radiologists have been dedicated to maintaining and continuously improving imaging protocols [6].

In this endeavor radiologists have always closely cooperated with many other scientists, including clinical physicists, MR physicists, IT professionals, and image processing specialists. Many of the technological advancements as well as optimization would not have been possible without this decade-long cooperation. Indeed, some of these related professions are presently fully integrated within large radiological departments.

\section{The advantage of organizational integration of all imaging services}

Referring physicians and patients expect the delivery of optimized imaging services. Factors to consider include the availability of best possible equipment, quality and assurance systems for imaging equipment, room design, patient communication, informed consent, patient transportation, patient surveillance, all aspects of timeliness, standardized (possibly evidence-based) protocols, professional communication, after-hour service, and emergency and disaster preparedness $[4,21]$. An integrated comprehensive management and organizational structure of imaging services has clear advantages and is better equipped to meet such high demands.

\section{Standardized workflow}

The workflow of an imaging service includes monitoring of the appropriateness of referral, quality assurance for professional and technical staff, report generation, archiving, and last but not least supervision and consultation by highly trained super-specialized radiologists (Fig. 1, adapted from [6]). For some parts of the process, information technology solutions may be beneficial and already available or under development [electronic order entry, automated decision support, computer aided detection (CAD)] [6].

\section{Quality and safety issues}

In radiology, the safety of patients and health care personnel permeates most features of the imaging process. According to Hendee, "it includes using the lowest dose of ionizing radiation possible to either achieve the images necessary to arrive at a correct diagnosis or conduct an interventional procedure successfully. Each image should be obtained at the lowest dose consistent with sufficient image quality, a minimum number of images should be

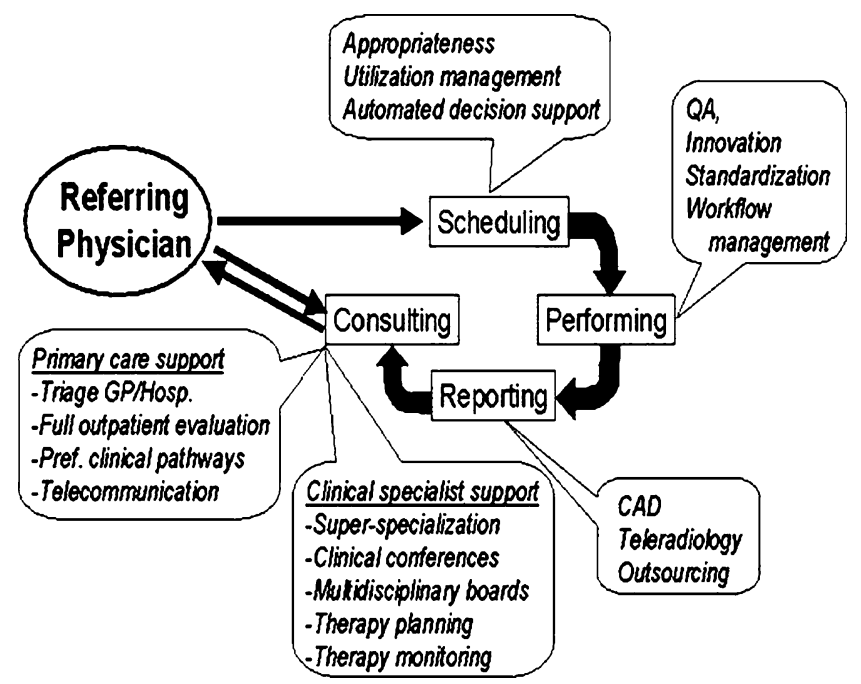

Fig. 1 The imaging workflow. Adapted, with kind permission from the Radiological Society of North America, from [6].

acquired consistent with the successful completion of an examination or procedure, and requests for unnecessary or inappropriate examinations should be refused. Every radiologic examination-even those that are performed with US and MR imaging, which do not employ ionizing radiation - expose patients to some element of risk. That risk comes from unwarranted exposure to radiation, as well as from false-positive results that lead to follow-up procedures and false-negative results that fail to demonstrate evidence of disease and injury. Knowledge about why, when, where, how, and for whom imaging should be employed is part of each radiologist's training" [1].

Thus a quality radiology practice will consistently perform the right procedure at the right time for the right patient, the radiology report will be timely and accurate, and the patient will receive optimal personal care $[4,6,21]$. However, this ideal is not reality, and even $99.99 \%$ reliability in a large practice will result in a significant number of adverse events [4]. Accountability of radiology departments is granted by the provider-customer relationship between referring physician and radiologist. Optimization of quality and safety requires a proactive systematic study of workflow, identification of weaknesses that could lead to suboptimal performance, and risk assessment [4]. Certainly, many of the well-known problems in radiology, such as lost or unavailable images and reports, delayed communication of results, and lack of patient information, have all been reduced with implementation of hospital and radiology information systems, voice recognition dictation systems, and picture archiving and communication systems. The quality of care and service has increased, and operational costs have decreased [22].

Quality standards should also assist in determining the technical specifications of new equipment being purchased, 
based on the facility's clinical imaging requirements. Guidelines should cover the general requirements specified for equipment selection with a view (1) to upgrading or maintaining standards for diagnostic imaging quality, i.e., the system design, construction, and performance, (2) to the extent and cost of service contracts and qualifications and availability of service personnel, and (3) to the cost of the system, system components and ancillary equipment as well as the cost of delivery and installation [23].

\section{4/7 services}

Increasingly, customers of imaging services look for timeliness and access [6]. Success of an integrated, comprehensive practice of imaging is related to a "365 by 24" (all day, every day) service that is not only valued but also needed by most referring physicians. In order to provide this continuous service, radiology needs a sufficient flow of patients in all possible technologies and indications also during office hours, particularly for training purposes and preserving expertise for emergency situations [6].

The large variety of procedures and the high levels of expertise available in subspecialized services have to be available also and particularly for emergency situations. For such levels of coverage, a minimum number of trained staff has to be available. This infrastructure is greatly appreciated by nonradiologist physicians and by health service administrators. The costs, however, may be substantial and a potent incentive for consolidated radiology departments.

\section{Decentralized technology}

An integrated comprehensive imaging service does not exclude the possibility of decentralizing some of the facilities (equipment) within the hospital [6]. Medicine will begin to take advantage of anytime/anywhere image interpretation enabled by digital acquisition and transmission [24]. Patient-focused decentralized care provides that patients in a given department rarely need to leave their hospital floor, as most of their needs (including administrative and discharge procedures, nutrition, patient support, and laboratory and simple radiographic services) are available on the floor. This method relies on decentralization of services by using mobile X-ray or ultrasound equipment handled by trained professionals belonging to the central imaging facility. Similarly, for image-guided interventions that have to be performed in special locations (operating theaters), radiographers and radiologists would follow the needs of the patient [6].

However, decentralization in radiology is wasteful and inefficient. It can make radiology departments smaller and less stimulating and employees less committed. When specialized work tasks are decentralized and stability in the work force is low, the risk is that specialized work tasks will not be done well. In a central department, technologists usually cover for each other during temporary absences and consult over technical problems. In the decentralized scheme, the technologist is isolated and works without direct supervision [6].

Radiology is a high efficiency, high throughput service. The large volume of procedures as well as clustering of radiological equipment allows optimized use of the scarce trained paramedical and medical personnel. Moreover, more equipment in the same modality offers the possibility of successive investment, allowing for the use of state-ofthe-art technology whenever necessary. These advantages are not available for small decentralized facilities [6].

\section{Internal competition}

Legislation in a free-market economic system does not tolerate attempts by entities to restrain or limit competition among providers of goods or services. Accordingly, a restraint or limitation on competition generally will be allowed only if it serves some important purpose. Over the years, health care organizations have recognized that a limitation on competition is acceptable if it protects a party from unfair competition. In this context, self-referral can be regarded as unfair internal competition with regard to services provided by a referralbased radiology department. Individual and interdepartmental economic competition and new technologies may renew competitive forces to obtain leadership positions and market share. These forces can become overwhelming and may stimulate detrimental competition for patients, space, and resources [6]. Moreover, internal competition among specialists interested in imaging may result in disagreements and may even begin to strain normally collegial relationships. On the other hand, a mutually beneficial arrangement among the specialties requires minimization of internal competition as well as adequate patient volume and economic resources for all participants. Competition must be therefore based externally on quality and cost, not internally among members of the group [25].

\section{Possible solutions}

"If we want things to stay as they are, things will have to change."

-Giuseppe di Lampedusa, The Leopard

The solutions imply that there is wide agreement on a number of starting principles concerning the integrity of radiology as a specialty and particularly the organizational integrity of all imaging services within the enterprise 
(hospital, multispecialty practice). As always and as previously stated in this paper, historically grown as well as efficiency-driven exceptions have to be part of the general agreement. Moreover, the presented solutions underscore clearly that diagnostic imaging and imageguided interventions are the domain of radiology and nuclear medicine, while collaboration with clinical specialties is frequently beneficial and needed. However, the integrity of the organizational structure of the "discipline of imaging" within the enterprise bears numerous benefits for cost-containment, quality standards, and efficiency [6].

The starting points are as follows:

1. Professional integrity of the specialty of radiology remains the leading principle. Radiologists and nuclear physicians are primarily responsible and in charge of performing all imaging procedures. Their expertise in collaboration with related professionals (clinical physicists, radiochemists, etc.) guarantees quality and safety, efficiency, and to the extent possible, evidence-based use of diagnostic imaging and image-guided interventions. It is preferable that the presently distinct specialties of radiology and nuclear medicine be brought together under one organizational umbrella, i.e., the imaging service. Exceptions for performing some specific procedures outside the departments of radiology or nuclear medicine or the joint image service are based on mutual agreements between the imaging and the clinical department.

2. Collaboration with other clinical specialists on an equal basis can be sought whenever there is evidence for qualitative improvement of patient care. Such improvement can be based on the clinical expertise of the involved specialist, the development of imaging expertise by other specialists, and - in limited situationsefficiency. For such a unified facility to function well and safely, the specialists will need to establish and maintain their imaging expertise. For some nonradiologist specialists, specific imaging is already included as part of their training (gastroenterology with abdominal/ endocavity ultrasound, cardiology with catheter angiography and interventions, or echoangiography). Clearly, these specialists in training need access to the equipment and have to perform a number of procedures in order to achieve credentialing. For those specialties that do not certify physicians for imaging but may have an added value for imaging, for example neuroscientists, another method of certification will need to be developed. One possibility would be to have the local radiologist community provide training and certification on an individual basis [6].

3. Imaging services are delivered and managed by a centralized comprehensive organization. Rules and quality standards in delivering services are similar throughout the whole organization. Part of these standards can be seen in the provision of double reading and expert supervision or application of standardized appropriateness criteria for referral and examination protocols. Exceptions are possible for a very limited number of specific procedures based on mutual agreement.

4. For establishment of a comprehensive electronic patient record, long-term availability of all imaging data is a necessity. The technical solution is based on an enterprise-wide PACS to which all providers of imaging services have to be connected and committed to similar workflow (electronic order entry, DICOMcompatible image acquisition and storage, electronic reporting, etc.). The enterprise-wide PACS is a solution managed primarily by the department of radiology or the joint imaging service in conjunction with the IT department of the enterprise which guarantees quality standards and availability of imaging data to all authorized users in the enterprise.

\section{Recommendations}

The future organizational position of diagnostic imaging and image-guided interventions in health-care enterprises has to take into account two specific aspects: integrity of diagnostic and interventional radiology as a clinical specialty and management of enterprise-wide imaging services.

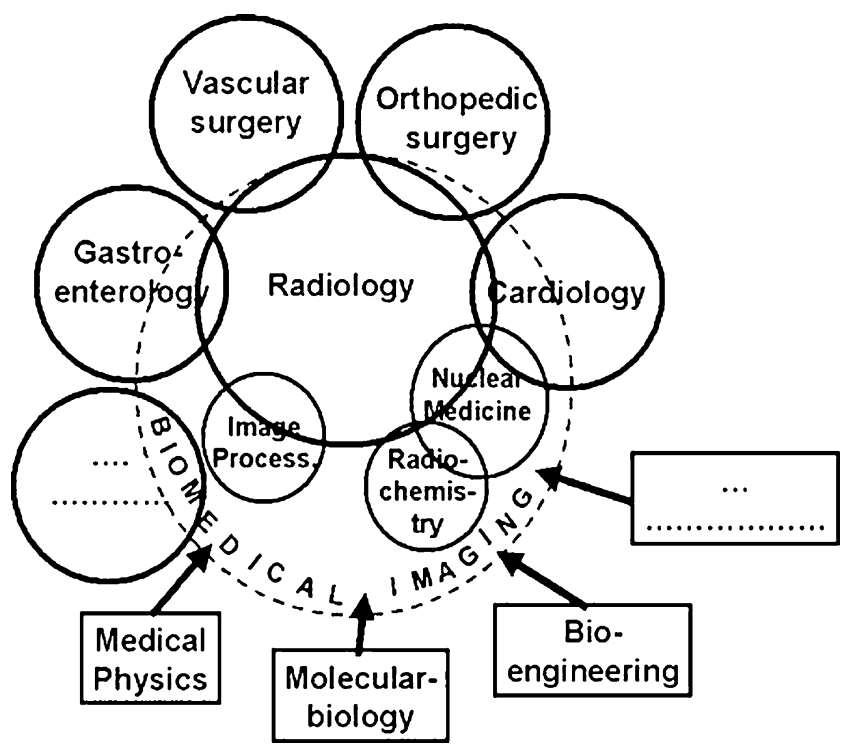

Fig. 2 The centralized imaging facility. Adapted, with kind permission from the Radiological Society of North America, from [6]. 
Integrity of diagnostic and interventional radiology as a clinical specialty

Radiology strives towards preservation of the integrity of the profession as a distinct clinical specialty. Large examination volumes and extended collaboration with clinical specialists within multidisciplinary groups allows for high quality subspecialized training of radiologists for different organ systems and diseases. System- or diseasebased radiologists should take the lead in innovation, development, and validation of new imaging technologies. The aim of radiologists is to serve the needs of the referring physicians by delivering high-quality examinations and reports and particularly by providing highly specialized consulting services. For historical and practical reasons, some of the areas of diagnostic imaging and image-guided interventions are provided directly by clinical specialists including dedicated ultrasound procedures or cardiac catheterization. In other instances, X-ray fluoroscopy is made available for diagnostic and particularly image-guided intervention purposes under the management of the radiology department and assistance of paramedical personnel, however, without involvement of radiologists. Such limited decentralized services are based upon mutual agreement between different clinical departments and the department of radiology. Finally, in some specific areas such as noninvasive cardiac imaging and endovascular interventions, a far-reaching collaboration between radiologists and clinical specialists on an equal basis seems to be the best approach for delivering high-quality patient care. Nuclear physicians deliver additional diagnostic imaging services as a second distinct medical imaging specialty. Despite the emergence of hybrid imaging modalities (PETCT, SPECT-CT, MR-PET), nuclear medicine remains for the time being a separate specialty. There are Europe-wide as well as national trends to re-merge radiology and nuclear medicine into one imaging specialty in the future; however, the discussion of this issue is outside the scope of this paper.

\section{Management of enterprise-wide imaging services}

From a management and organization point of view, a unified and centralized structure including all imageproducing and related specialties into one single enterprise-wide "imaging facility" seems beneficial (Fig. 2, modified from [6]). Such an approach would guarantee comparable workflow with standardized referral procedures, acquisition protocols, data storage, image postprocessing, image interpretation, and consulting services. Such a centralized organization would also guarantee high quality equipment operation by trained paramedic personnel, quality assurance and maintenance by clinical physicists and medical technicians.
Acknowledgements This paper expresses continuing thoughts on concepts presented previously in: Krestin GP (2009) Maintaining identity in a changing environment: the professional and organizational future of radiology. Radiology 250(3):612-617. Paper prepared by Gabriel P. Krestin. Approved by the ESR Executive Council, June 2009.

Open Access This article is distributed under the terms of the Creative Commons Attribution Noncommercial License which permits any noncommercial use, distribution, and reproduction in any medium, provided the original author(s) and source are credited.

\section{References}

1. Hendee WR (2006) An opportunity for radiology. Radiology 238:389-394

2. Schnyder P, Capasso P, Meuwly JY (1999) Turf battles in radiology: how to avoid / how to fight / how to win. Eur Radiol 9:741-748

3. Margulis AR, Sunshine JH (2000) Radiology at the turn of the millennium. Radiology 214:15-23

4. Miller JC, Thrall JH, Golding SJ, Frija G, Glazer GM, Ringertz HG, Krestin GP (2005) Re-inventing radiology in a digital and molecular age. Summary of proceedings of the 6th bi-annual symposium of IS ${ }^{3} \mathrm{R}$, August 25-27, 2005

5. Alderson PO (2000) A balanced subspecialization strategy for radiology in the new millennium. AJR Am J Roentgenol 175:7-8

6. Krestin GP (2009) Maintaining identity in a changing environment: the professional and organizational future of radiology. Radiology 250:612-617

7. Pigache P (2007) Radiology turf wars: is there a roadmap for peace? HIRE 2(3):11-13

8. Levin DC, Rao VM (2004) Turf wars in radiology: the overutilization of imaging resulting from self-referral. J Am Coll Radiol 1(3):169-172

9. DeMaria AN (2005) The growth of diagnostic imaging services. J Am Coll Cardiol 45(12):2093-2094

10. Becker GJ (2001) The future of interventional radiology. Radiology 220(2):281-292

11. Rostenberg B (2007) 'Surgology' is coming! Designing for the convergence of surgery and interventional radiology. Health Facil Manage 18(6):49-52

12. Levin DC, Abrams HL, Castaneda-Zuñiga WR et al (1994) Why radiologists lost coronary angiography and what can be done to prevent future similar losses. Invest Radiol 29(4):480-484

13. Levin DC, Rao VM, Bonn J (2005) Turf wars in radiology: the battle for peripheral vascular interventions. J Am Coll Radiol 2:68-71

14. Enzmann DR (2005) A different look at turf. Radiology 234:347-349

15. Veith FJ, Marin ML (1995) Endovascular surgery and its effect on the relationship between vascular surgery and radiology. J Endovasc Surg 2:1-7

16. Porter JM (1990) Vascular surgery residency training curriculum. J Vasc Surg 12:79-83

17. Hall FM (1988) Turf battles in interventional radiology. AJR Am J Roentgenol 151:1247-1248

18. Veith FJ, Marin ML (1996) Endovascular technology and its impact on the relationships among vascular surgeons, interventional radiologists and other specialists. World J Surg 20:687-691

19. Dalla Palma L (2006) Tomorrow's radiologist: what future? Radiol Med 111:621-633

20. Hillman BJ, Joseph CA, Mabry MR, Sunshine JH, Kennedy SD, Noether M (1990) Frequency and costs of diagnostic imaging in office practice: a comparison of self-referring and radiologistreferring physicians. N Engl J Med 323:1604-1608 
21. Thrall JH (2004) Quality and safety revolution in health care. Radiology 233:3-6

22. Thrall JH (2005) Reinventing radiology in the digital age part I. The all-digital department. Radiology 235:382-385

23. Périard MA, Chaloner P (1996) Diagnostic X-ray imaging quality assurance: an overview. Can J Med Radiat Technol 27:171-177
24. Thrall JH (2005) Reinventing radiology in the digital age part III. Facilities, work processes, and job responsibilities. Radiology 237:790-793

25. Zarins CK (1996) Unified multispecialty approach: is it a viable response to new technology used in the care of vascular patients? J Endovasc Surg 3:364-368 